\title{
CRISIS DEMOGRÁFICA EN LA SIERRA MORENA ONUBENSE *
}

Jesús MONTEAGUDO LOPEZ-MENCHERO** Juan MARQUEZ DOMINGUEZ***

\section{LA SIERRA EN LA COMARCALIZACION ONUBENSE}

Tradicionalmente se han distinguido en Huelva cuatro comarcas: Sierra, Andévalo, Campiña y Costa. Aunque esta comarcalización se ha modificado sensiblemente tras la instalación del Polo de Desarrollo, creándose nuevas comarcas en función del impacto directo de este foco industrial (Organización Sindical, FOURNEAU, MONTEAGUDO), sin embargo la Sierra continúa teniendo la misma personalidad.

GONZALO Y TARIN, ya en 1886, en sus "comarcas naturales" trataba de las divisiones de Huelva. Una de ellas era la Septentrional o región montañosa, formada por las derivaciones meridionales y occidentales de Sierra Morena. En este trabajo nos vamos a circunscribir a la denominada por él, Sierra alta o de Aracena.

La inalterabilidad territorial de esta comarca se debe al mantenimiento de una economía tradicional y al predominio del medio físico en los factores de producción, frente a lo ocurrido en los sectores meridionales de la provincia, en torno a la capital, donde unos municipios se han segregado de "comarcas naturales" y jos referidos a ella tomen siempre un límite físico, el escalón de Sierra Morena respecto al piedemonte del Andévalo (AVILA, 1982).

$\left(^{*}\right)$ Comunicación presentada al Congreso sobre Agricultura y Desarrollo Rural en Zonas de Montaña. Granada, 1985.

${ }^{* *}$ ) Profesor Titular de Geografía Humana. Colegio Universitario de La Rábida, Univ. de Sevilla.

(***) Profesor de Análisis Geográfico Regional. Colegio Univ. de la Rábida, Univ. de Sevilla. 


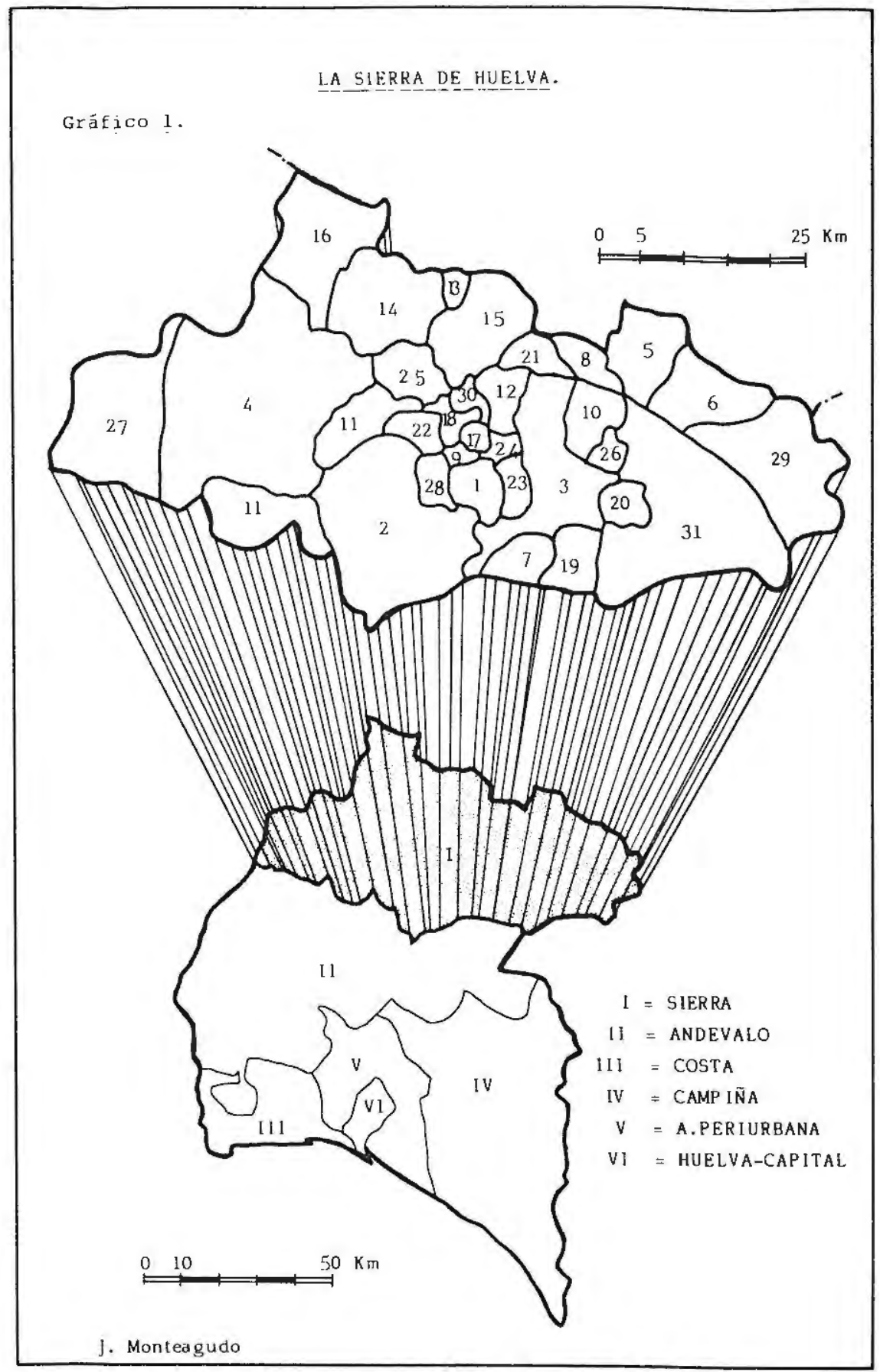


Se constituye por lo tanto en el sector más septentrional de la provincia de Huelva. Abarca administrativamente 31 municipios y un partido judicial, el de Aracena. Se extiende a lo largo de $3.079,47 \mathrm{~km}^{2}$, que representan el 31 por 100 de la superficie provincial, siendo la extensión media por municipio de 99,34 $\mathrm{km}^{2}$. Existe en este sentido una notable diferencia entre unos y otros, como por ejemplo Aroche $\left(498,39 \mathrm{~km}^{2}\right)$ y Los Marines $\left(9,89 \mathrm{~km}^{2}\right)$. Estas diferencias de extensión tendrán indudable influencia sobre la población, puesto que son actividades agropecuarias la principal fuente de riqueza, y el espacio el factor esencial de ellas.

\section{RASGOS ECONOMICOS GENERALES DE LA SIERRA DE HUELVA}

Este espacio provincial destaca físicamente por su mayor altitud que el resto de la provincia, lo que le permite alcanzar una mayor pluviosidad que unida a las levemente menores temperaturas, provoca un grado de humedad más elevado que en los demás sectores provinciales. Sus materiales son antiguos, en general esquistosos, con afloramientos de calizas y granito en sectores concretos. Son por tanto rocas de difícil alterabilidad y por ello las únicas tierras potencialmente útiles para el cultivo son los fondos de los valles rellenos con aportes sedimentarios de los sectores más elevados. En general predominan litosuelos de difícil cultivo y bajo rendimiento. A pesar de esta aparente adversidad física, la economía serrana se basa fundamentalmente en el aprovechamiento de los recursos que le brinda este medio. Sobre este soporte se asientan montes de encinas y alcornoques adehesados. Cuando un espacio cultivado se abandona es ocupado inmediatamente por un matorral formado por la diversidad de especies que conforman el sotobosque mediterráneo. Este matorral, por la abundancia de tales terrenos y el progresivo abandono de las dehesas, va siendo cada vez más frecuente en Huelva.

Por todas estas razones la economía serrana es netamente agropecuaria y forestal. La superficie censada es de $287.373 \mathrm{Ha}$. que representan el 93,3 por 100 de la superficie geográfica de la comarca. De esa superficie censada, $32.838 \mathrm{Ha}$. son tierras labradas, el 11,4 por 100 de las censadas. Puesto que en el conjunto provincial la proporción de tierras labradas respecto al total de superfície censada es del 18 por 100, y si se considera el espacio provincial menos el serrano la proporción asciende al 20,1 por 100 , se puede situar el área serrana dentro del marco de las economías agrarias marginales, condicionado su sistema agrario por la influencia excesiva del medio físico. 
De esa superficie labrada se destinan a cultivos herbáceos $9.566 \mathrm{Ha}$. que representan el 29,1 por 100. Esta cifra contrasta con la proporción que a dichos cultivos se destina en el conjunto provincial, el 54,5 por 100 , lo que denota la escasa potencialidad serrana para este tipo de cultivos. En todo caso los cultivos herbáceos aparecen asociados a especies forestales, lo que les confiere un claro carácter de funcionalidad ganadera; son en general forrajeras y pratenses y ocupan el 32,1 por 100 de la superficie serrana labrada, mientras que en el conjunto provincial la alternancia herbáceos-forestales se da sólo en el 8,3 por 100 de la superficie labrada. Incluso puede reforzar esta vocación alternante de la Sierra el hecho de que sus $10.521 \mathrm{Ha}$. de herbáceos con especies forestales, son el 70,9 por $100 \mathrm{de}$ las $14.837 \mathrm{Ha}$. de las mismas características en toda la provincia.

El olivo sí es una especie extendida por la Sierra. El 36,2 por 100 de su superficie labrada está ocupada por él, solo o asociado con herbáceos. En la provincia su representación se reduce al 17,3 por 100 de la superficie labrada. Finalmente el viñedo sólo ocupa el 0,3 por 100 de las tierras de labranza y los frutales el 2,3 por 100 . En el conjunto provincial ocupan ambas especies el 7,9 y 8,5 por 100 respectivamente.

Se deduce fácilmente que la Sierra no destaca por su economía agrícola, ya que incluso los cultivos más representativos como es el caso del olivar, se encuentran en franca regresión debido al encarecimiento de la mano de obra y a los impedimentos que la topografía impone a la mecanización (J. MARQUEZ). Los frutales y hortalizas quedan reducidos a los ruedos de algunos municipios del área de Aracena (Alájar, Castaño, Fuenteheridos, Galaroza, Jabugo, Los Marines, Valdelarco).

A estos aspectos de distribución espacial de los aprovechamientos hay que añadir dos: el primero es la menor rentabilidad de estos cultivos en la Sierra que en otras comarcas, puesto que sus rendimientos son en general inferiores a la media provincial; el segundo es el desigual tamaño de las explotaciones, ya que dada la general escasa calidad de los suelos, las explotaciones agropecuarias y forestales son extensas y las tierras labradas quedan atomizadas en multitud de pequeñísimas explotaciones, dispersas incluso las de un mismo propietario, que confieren a la empresa una escasísima rentabilidad y las reduce casi al autoconsumo. Una muestra de ello es que el tamaño medio de las explotaciones en la Sierra es de 55,3 Ha.; de su superficie labrada la media es de 7,5 Ha. y de la no labrada de $310,8 \mathrm{Ha}$.

Por estas características de la agricultura, la ganadería se convierte en la principal protagonista de la actividad agraria. Acoge a 19.078 cabezas de ganado bovino (51,6 por 100 del bovino provincial), 43.358 cabezas de ovino (22,5 por 100 provincial), 31.020 cabezas de caprino (50,8 рог 100 provincial), y, sobre todo, 63.491 cabezas de porcino (64,6 por 100 provincial), cuya importancia trasciende 
de la cantidad para centrarse en la calidad. Se trata del cerdo ibérico cuyos productos derivados dan renombre a la subcomarca de Jabugo. Esta producción porcina se ve cada vez más disminuida tanto por la continua agresión de la peste porcina africana, que diezma la cabaña, como por el incremento de los costes de producción y la competencia de especies forestales alóctonas que merman el espacio adehesado, dedicado tradicionalmente a la actividad silvopastoril.

Todo esto va unido a un régimen en el que predomina la explotación directa por el propietario ( 72,4 por 100 de las tierras), bien como obrero-empresario ayudado por su familia, bien por medio de obreros agrícolas, jornaleros y temporeros, en las explotaciones de mayor tamaño. Unicamente un 14,4 por 100 de la superficie está arrendada y un 1,1 por 100 tiene un régimen de aparecería. El 12,1 por 100 tiene otros regímenes diversos.

Inmersas en esta estructura socio-jurídica agraria se encuentran las 4.029 Unidades de Trabajo Agrario al año, que suponen 0,014 U.T.A. por Ha. y 0,12 U.T.A. por Ha. si sólo se consideran las tierras labradas. En el conjunto de la provincia los valores son 0,017 y 0,09 U.T.A.-Ha. respectivamente. La primera cifra no hace sino traducir la importancia que adquieren en la Sierra las tierras no labradas y la escasa ocupación en ellas. La segunda puede explicarse por alguna de estas razones o por la conjunción de todas ellas: a) Existencia de un excedente de población agraria. b) Las dificultades topográficas impiden mecanizar los cultivos. c) La peculiar simbiosis agricultura-ganadería-bosque demanda más mano de obra que el simple sector agrícola.

Como comentábamos anteriormente, hay que destacar la importancia que adquiere el trabajo procedente de las ayudas familiares, el 17,8 por 100 del total serrano, frente al 13,1 de la media provincial. El trabajo empresarial representa el 33 por 100 de las U.T.A., mientras que el trabajo asalariado es el 49,2 por 100 . Se deduce un claro predominio del trabajo asalariado repecto al empresarial, que incluso es mayor del que las cifras ofrecen puesto que numerosos empresarios y ayudas familiares trabajan a jornal cuando existe posibilidad.

A estos aspectos hay que añadir la venta sin apenas transformación "in situ" del corcho, que genera todas las plusvalías fuera del espacio onubense; la producción de castaña, otro recurso importante, que se encuentra atomizada tanto en su producción como en su comercialización e impide al agricultor obtener unos beneficios que revierten en manos de mayoristas e intermediarios; la creación, en los primeros años de la década de 1960, de una fábrica de celulosas en Huelva que demanda materia prima y ha producido una de las mayores transformaciones del paisaje onubense en general, cambiando terrenos forestales, dehesas y otros cultivos, en continuos eucaliptales (D. MARQUEZ). 
A estos términos estructurales hay que añadir dos coyunturales. Uno se produce fuera de este ámbito con resultados negativos para el mantenimiento de la población en el área. Se trata de la creación del Polo de Desarrollo en Huelva y el traslado de establecimientos industriales de la cuenca minera del Andévalo hacia el área de Huelva, con la consiguiente succión de población que esto provoca. Será uno de los motores del éxodo rural de la Sierra. Otro, positivo, es el que está teniendo lugar en la actualidad y que consiste en la potenciación de los recursos paisajísticos y medioambientales con fines turísticos. Tal atención podría generar nuevas espectativas para la población de estos municipios y contribuiría a fijarla y dotarla de mejor nivel de vida, si se viese apoyada tanto por la creación de una infraestructura viaria, que terminase con el secular aislamiento en que se ha visto siempre esta comarca, como por la dotación de servicios sociales en su espacio.

Los indicadores socioeconómicos generales expresan esta situación marginal. BANESTO en su estudio sobre la renta por habitante de los municipios españoles, señala que todos los serranos menos seis (Cumbres de Enmedio, Cumbres Mayores, Jabugo, Castaño, Aracena e Higuera) tienen rentas entre $170.001 \mathrm{y}$ 230.000 pesetas; los citados seis están en el siguiente escalón de 230.000 a 280.000. Ninguno alcanza los dos últimos tramos que propone BANESTO: hasta 500.000 pts. y más de 500.000 , aunque en esta última escala no existe ningún onubense.

Por otra parte, de una relación entre el presupuesto municipal y el número de habitantes (cuadro I), se puede deducir la pobreza de los municipios serranos onubenses, incluso en relación con el resto de la provincia. En efecto, la media de presupuesto/habitante es de 8.598 pesetas, cuando en el conjunto provincial es de 10.670. Significa esto que sus recursos municipales están muy por debajo de la media provincial. Incluso, como puede apreciarse en el cuadro I, existen tres municipios que no alcanzan ni las 5.000 pts./hab. De los 31 municipios, 11 superan la media, pero sólo dos de ellos están por encima de la media provincial.

En definitiva, estamos ante un espacio falto de recursos, con una actividad agropecuaria que no es suficiente para soportar el peso demográfico de la Sierra, y, paradójicamente, para poder mantener este tipo de actividad agropecuaria y forestal necesita enviar población fuera de su espacio. Por este motivo la pérdida constante de población es una de las características de la demografía serrana. 


\section{CUADRO I}

VALORES PRESUPUESTARIOS DE LA SIERRA DE HUELVA

Municipios

1. Alájar

2. Almonaster la Real

3. Aracena

4. Aroche

5. Arroyomolinos de León

6. Cala

7. Campofrío

8. Cañaveral de León

9. Castaño del Robledo

10. Corteconcepción

11. Cortegana

12. Cortelazor

13. Cumbres de Enmedio

14. Cumbres de San Bartolomé

15. Cumbres Mayores

16. Encinasola

17. Fuenteheridos

18. Galaroza

19. Granada de Riotinto, La

20. Higuera de la Sierra

21. Hinojales

22. Jabugo

23. Linares de la Sierra

24. Marines, Los

25. Nava

26. Puerto Moral

27. Rosal de la Frontera

28. Santa Ana la Real

29. Santa Olalla del Cala

30. Valdelarco

31. Zufre

Sierra de Huelva

Población

937

2.469

6.276

4.013

1.314

1.546

932

636

251

761

5.684

443

66

803

2.585

2.659

687

1.823

233

1.497

546

2.534

322

387

402

236

2.701

648

2.362

326

2.043

47.489
Presupuesto

Pres./Hab.

8.004

7.914

19.539 .438

16.093

9.690

6.545

6.382

5.593

5.213 .000

3.616

2.300 .000

6.494

5.500 .000

7.227

37.052 .254

6.519

4.515 .000

10.192

529.195

8.018

4.500 .000

5.604

26.107 .718

10.100

18.800 .000

7.070

8.443

6.583

12.000 .000

6.009

1.400 .000

3.839

3.846

2.100 .000

12.156

30.802 .615

9.472

3.050 .000

8.527

2.761.869

6.870

1.241.474

5.260

7.726

7.048

6.189

5.726

5.628

11.497 .682

8.598

Fuente: I.N.E. y Memoria de la Cámara Of de Comercio. Ind. y Nav. de Huelva. Año 1984. Población y presupuestos corresponden a 1984.

Elaboración propia. 


\section{LA POBLACION DE LA SIERRA}

Hemos tomado la fecha de 1900 , inicio del siglo, para analizar el proceso evolutivo de la población de este área, proceso que culminamos en los últimos datos oficiales publicados, los del Censo de 1 de marzo de 1981.

Tomando como base el año 1900, el gráfico 2 permite observar la evolución del índice de la Sierra. Asimismo se traducen en él la escasa densidad de población, $15 \mathrm{~h} / \mathrm{km}^{2}$ en 1981, y el poco peso que esta población representa en el conjunto de la provincia a pesar de agrupar al 31 por 100 de su superficie. También se puede observar que su descenso se inicia destacadamente después de 1960, siendo las décadas 1960-1980 las más negativas de esta comarca.

\begin{tabular}{lcccc}
\hline Año & Población & Indice & Densidad & $\%\left({ }^{*}\right)$ \\
\hline & & & & \\
1900 & 63.178 & 100 & 20 & 24 \\
1910 & 73.115 & 116 & 24 & 24 \\
1920 & 75.805 & 120 & 24 & 22 \\
1930 & 77.334 & 122 & 25 & 20 \\
1940 & 75.015 & 119 & 24 & 20 \\
1950 & 73.626 & 117 & 24 & 18 \\
1960 & 74.211 & 117 & 24 & 15 \\
1970 & 60.272 & 95 & 19 & 11 \\
1981 & 45.589 & 73 & 15 & \\
\hline
\end{tabular}

Fuente: I.N.E. Elaboración propia.

(*) \% de población respecto a la provincia.

Por esta evidencia, que se manifiesta a partir de la década 1960-70, que coincide en España con el mayor éxodo rural, con el despegue industrial y con el mayor indice de mecanización del campo, y en Huelva con la creación de un Polo Industrial a mediados de la década, es por lo que hemos querido dividir el análisis de la población de la Sierra en tres grandes períodos: 1900-1950, coincidiendo con la mitad del siglo XX e inicio ya de un moderado éxodo rural; 1950-1965, período que finaliza con la creación del Polo en Huelva; y 1965-1981 que es la última fase de la que poseemos información.

Los cuadros II, III y IV apoyan este comentario.

En la primera mitad del siglo XX la Sierra gana 10.448 h., cifra que corresponde aproximadamente al mantenimiento del crecimiento natural en su lugar de origen. Durante este período 12 municipios tienen un crecimiento negativo y ninguno supera el -1 por 100 anual, aproximándose a esta cifra Castaño del Robledo. 


\section{INDICES,DENSIDAD Y PROPORCION DE POBLACION EN LA SIERRA DE HUELVA}

Gráfico 2 .

INDICES DE EVOLUCION DE LA POBLACION.

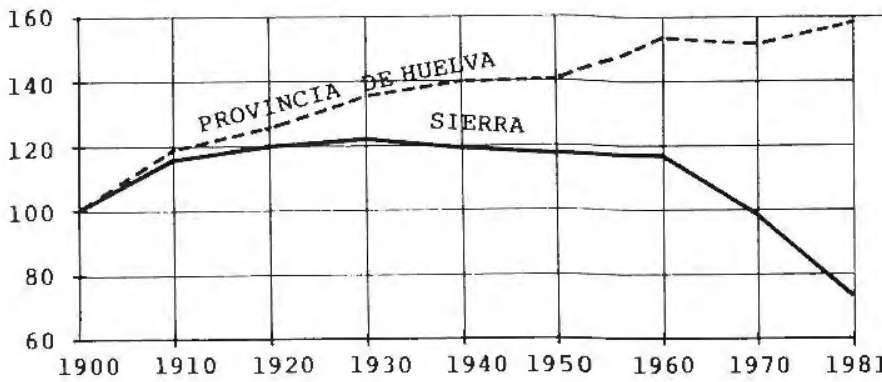

DENSIDAD DE POBLACION ( $\mathrm{H} . / \mathrm{KM})$.

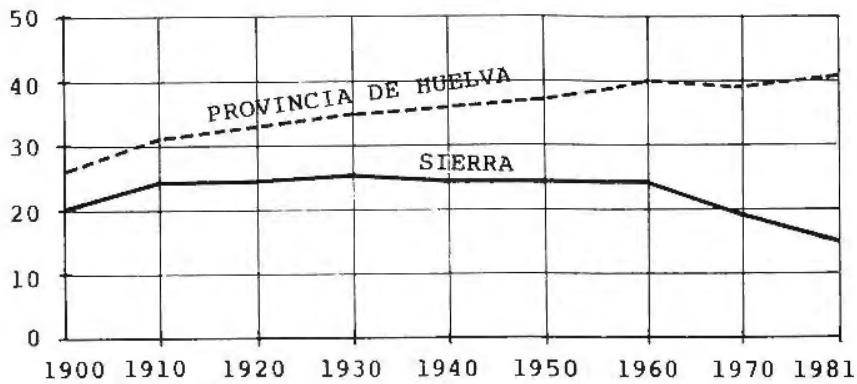

PROPORCION DE LA POBLACION SERRANA EN LA PROVINCIA.



Fuente: I.N.E.

J. Monteagudo. 
CUADRO II

EVOLUCION DE LA POBLACION EN LA SIERRA DE HUELVA

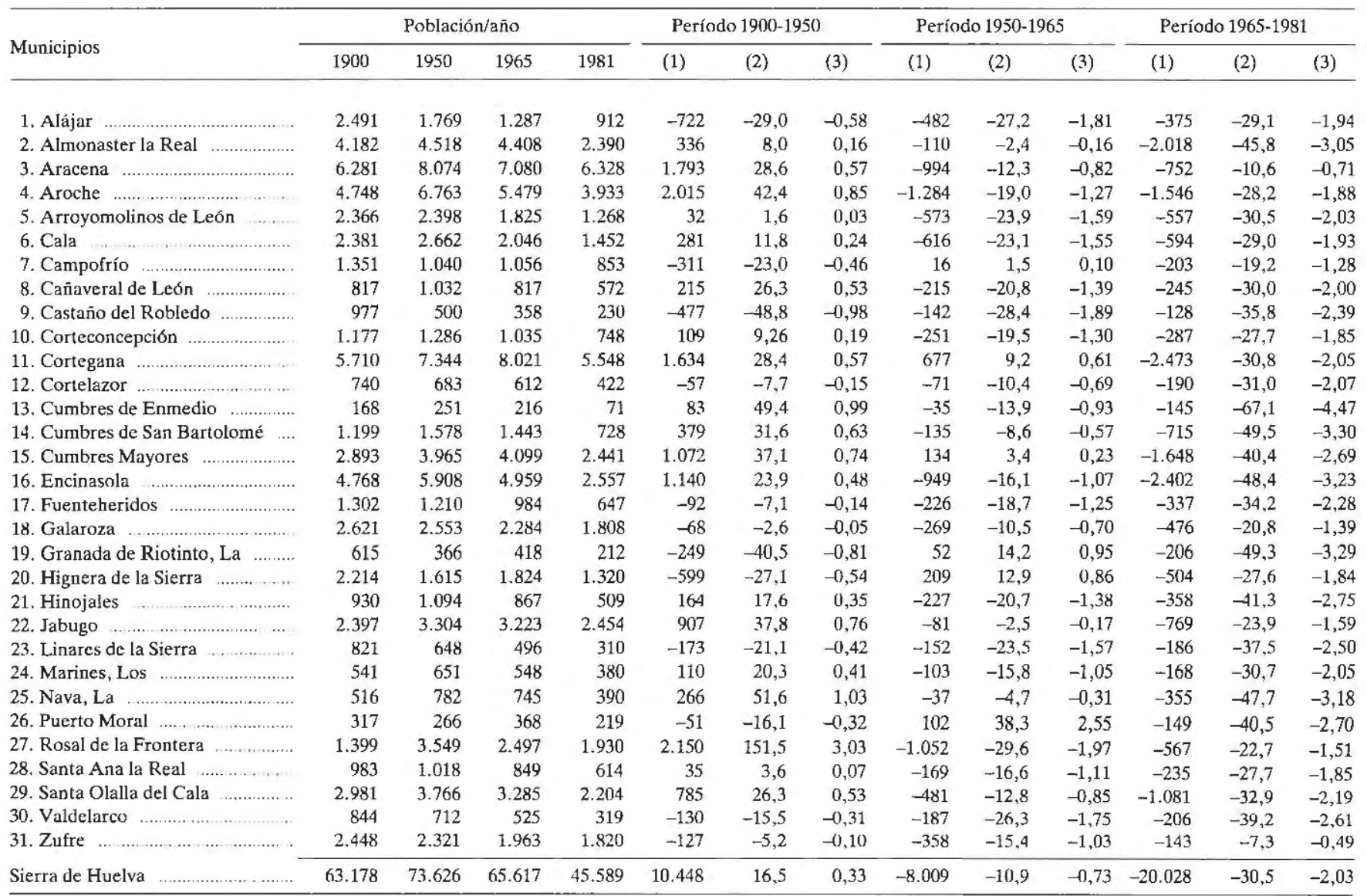

Fuente: I.N.E.

Elaboración propia.

(1) Diferencia $\mathrm{n} .{ }^{\circ}$ de habitantes. 


\section{EVOLUCION DE LA POBLACION DE LA SIERRA DE HUELVA.} VALORES ANUALES.

Gráfico 3 .
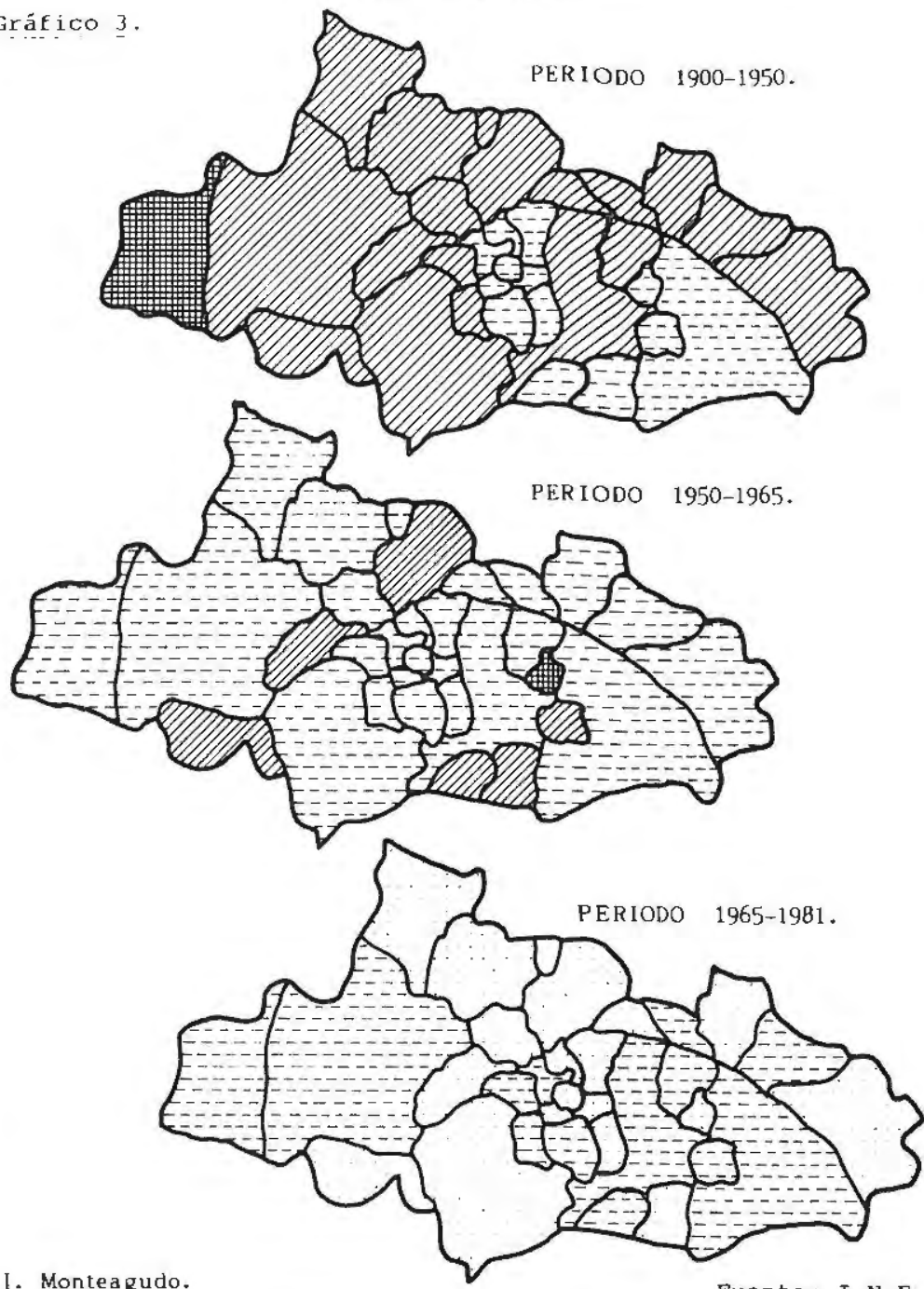

J. Monteagudo.

Fuente: I.N.E.
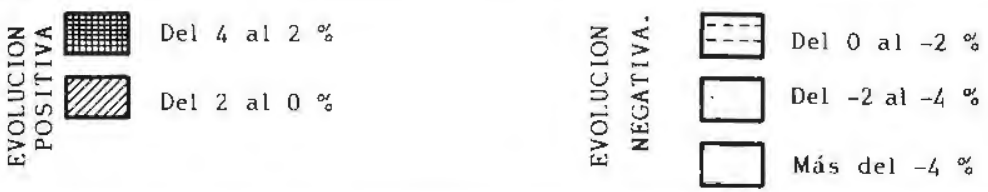
En el período 1950-1965 la población disminuye en 8.009 h. cifra importante si tenemos en cuenta que a éstos hay que añadir el crecimiento natural que se ha producido. En este período son ya 25 los municipios que pierden población y 16 de ellos superan el descenso del -1 por 100 anual.

En 1964 comienza en Huelva el Polo de Promoción Industrial y las comarcas de la provincia basculan hacia la capital y su entorno, produciendo una corriente migratoria que no cesa hasta 1975 , año en que el crecimiento industrial se detiene y comienza a hacer su efecto la crisis de 1973 . No obstante continuaba la corriente migratoria hacia la capital. En 1981, de los habitantes de Huelva-capital que no habían nacido en la ciudad, 4.623 lo habían hecho en los municipios de la Sierra y suponían el 16,4 por 100 de la inmigración a Huelva-capital. En este último período todos los municipios serranos pierden población y todos superan el -1 por 100 anual, excepto dos, llegando incluso al -4 por 100 como es el caso de Cumbres de Enmedio. En estos últimos 15 años la Sierra pierde 20.028 habitantes que representan un $-30,5$ por 100 .

La gravedad de esta pérdida masiva de población no está sólo en el número de quienes se van, sino en que los que emigran son los más productivos, los más jóvenes y emprendedores, haciendo válidas las tesis de Myrdal: la emigración se produce en zonas pobres porque no hay recursos suficientes y al emigrar los más productivos la pobreza es cada vez mayor.

La demostraeión de este hecho viene dada al menos en función de la estructura por edades de la población. Los menores de 16 años representan el 23,3 por 100, mientras que en el conjunto provincial son el 28,7 y en España el 25,6 por 100; es decir, existe una escasa población infantil, consecuencia del también corto número de población en edad de procrear. Los comprendidos entre 16 y 64 años representan un 58,4 por 100; en la provincia son el 60 por 100 y en España el 63,1 por 100. Queda patente no sólo el carácter emigratorio de la Sierra, sino también de la provincia de Huelva, con proporciones de población potencialmente activa inferiores a la nacional. Finalmente, los mayores de 65 años representan en la Sierra el 18,3 por 100 , cifra elevadísima si la comparamos con la provincia y la nacional, ambas el 11,3 por 100 . Es una muestra evidente del excesivo envejecimiento de la población serrana, algo que se traduce en la edad media de empresarios y asalariados agrarios.

A nivel municipal, como se puede observar en el cuadro III, son numerosos los municipios que no alcanzan el 20 por 100 de población infantil, llegando incluso Cumbres de Enmedio a no censar a nadie en esas edades. Por el contrario, son también numerosos los que rebasan el 20 por 100 de población mayor de 65 años, e incluso en dos ocasiones se rebasa el 30 por 100, casos de Castaño del Robledo $(30,8$ por 100$)$ y Cumbres de Enmedio (50,3 por 100$)$, dando muestras de un evidente abandono de la población potencialmente activa. 


\section{CUADRO III}

ESTRUCTURA DE LA POBLACION POR GRUPOS DE EDAD

Municipios

Grupos de edad (\%)

0-15 $16-64 \quad 65$ y más

1. Alájar

2. Almonaster la Real

3. Aracena

4. Aroche

5. Arroyomolinos de León

6. Cala

7. Campofrío

8. Cañaveral de León

9. Castaño del Robledo

10. Corteconcepción

11. Cortegana

12. Cortelazor

13. Cumbres de Enmedio

14. Cumbres de San Bartolomé

15. Cumbres Mayores

16. Encinasola

17. Fuenteheridos

18. Galaroza

19. Granada de Riotinto, La

20. Higuera de la Sierra

21. Hinojales

22. Jabugo

23. Linares de la Sierra

24. Marines, Los

25. Nava, La

26. Puerto Moral

27. Rosal de la Frontera

28. Santa Ana la Real

29. Santa Olalla del Cala

30. Valdelarco

31. Zufre

Sierra de Huelva
14,7

24,4

28,0

24,5

15,8

30,5

17,4

10,7

11,6

26,5

23,2

19,0

-

15,0

24,6

14,7

28.3

22,2

23,0

20,8

31,6

20,9

25,4

30,3

10,7

20,0

24,1

21,8

27,6

17,8

27,3
61,7

59,0

57,0

55,3

54,6

54,7

67,7

62,7

57,6

62,8

57,4

51,2

49,7

59,1

59,5

59,6

61,3

56,7

58,1

58,4

55,5

63,8

62,6

46,9

59,9

59,9

60,0

60,8

57,3

61,2

60,3

58,4
23,6

16,6

15,0

20,2

29,6

14,8

14,9

26,6

30,8

10,7

19,4

29,8

50,3

25,9

15,9

25,7

10,4

21, 1

18,9

20,8

12,8

15,3

12,0

22,9

29,4

20,1

15,9

17,4

15,1

21,0

12,4

23,3

18,3

Fuente: I.N.E.

Elaboración propia. 


\section{EL POBLAMIENTO SERRANO}

La desertización humana de la Sierra de Huelva no sólo se puede medir a través de la pérdida bruta de población y de la estructura por edades, sino que también es una muestra evidente de este hecho lo que sucede con el poblamiento. Esta pérdida masiva de población se produce primero en los núcleos más pequeños, en las aldeas, y progresivamente alcanza a la cabecera, de tal forma que muchos núcleos quedan literalmente abandonados y el poblamiento se va concentrando cada vez en menos núcleos. Pruebas evidentes son eI gráfico 4 y el cuadro IV, que reflejan la realidad serrana a través del índice de dispersión de Colas. Este índice se obtiene mediante una fracción en cuyo numerador figuran la superficie del espacio que se considere (S), en este caso el término municipal, multiplicada por el número de núcleos en diseminado $(\mathrm{N})$; y cuyo denominador es la diferencia entre la población total $(\mathrm{T})$ y la población en diseminado $(\mathrm{E})$; en definitiva, la población de la cabecera municipal. El inconveniente de este índice es que a igualdad de núcleos y población diseminada, la superficie puede dar la impresión de más dispersión o menos. Puesto que trabajamos con términos municipales, este hecho provocará que los mayores en superficie tiendan a representar mayor dispersión. A pesar de que esa superficie es arbitraria ya que se trata de circunscripciones administrativas, no puramente geográficas, el índice es válido y nos parece incluso más adecuado que el de Demangeon que no hace intervenir la superficie.

Al margen de todas las críticas que puedan hacerse a estos indicadores (véase SUAREZ JAPON), los vamos a utilizar aquí para comparar la situación serrana onubense en tres momentos, distanciados entre sí veinte años cada uno: 1940, 1960 y 1981. Nos basamos en los nomenclátores de esos años.

Se puede observar en el gráfico 4 que en 1940 sólo un municipio, Valdelarco, tiene un índice inferior a 0,01, es decir, un grado de concentración elevado. No sólo es de pequeña superficie, sino que además es el único que agrupa a toda la población en un solo núcleo, la cabecera. Frente a él otros como Almonaster la Real que superan el índice 10 , que indica tanto una elevada extensión como un alto número de núcleos dispersos, en el caso que apuntamos, 31. En este año son 10 los municipios que alcanzan al menos los 10 núcleos dispersos y 12 los que tienen menos de 5 núcleos.

En 1960 ningún municipio alcanza el índice 10 de dispersión, puesto que Almonaster ha descendido a 25 núcleos dispersos. Sólo 3 municipios tienen más de 10 núcleos dispersos y son ya 26 los que tienen menos de 5 núcleos dispersos, y de ellos 13 sólo tienen población en la cabecera. En estos veinte años se observa un claro abandono de entidades menores que van quedando destruidas o conservadas únicamente como parte de unidades de explotación, no como habitat permanente. 
DISPERSION DEL HABITAT EN LA SIERRA DE HUELVA. INDICE DE COLAS.

Grăfico 4 .

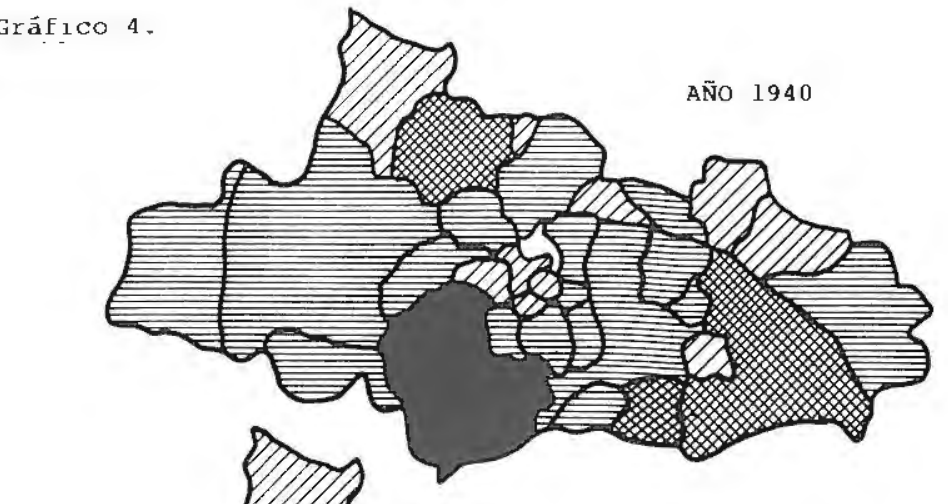

AÑO 1960
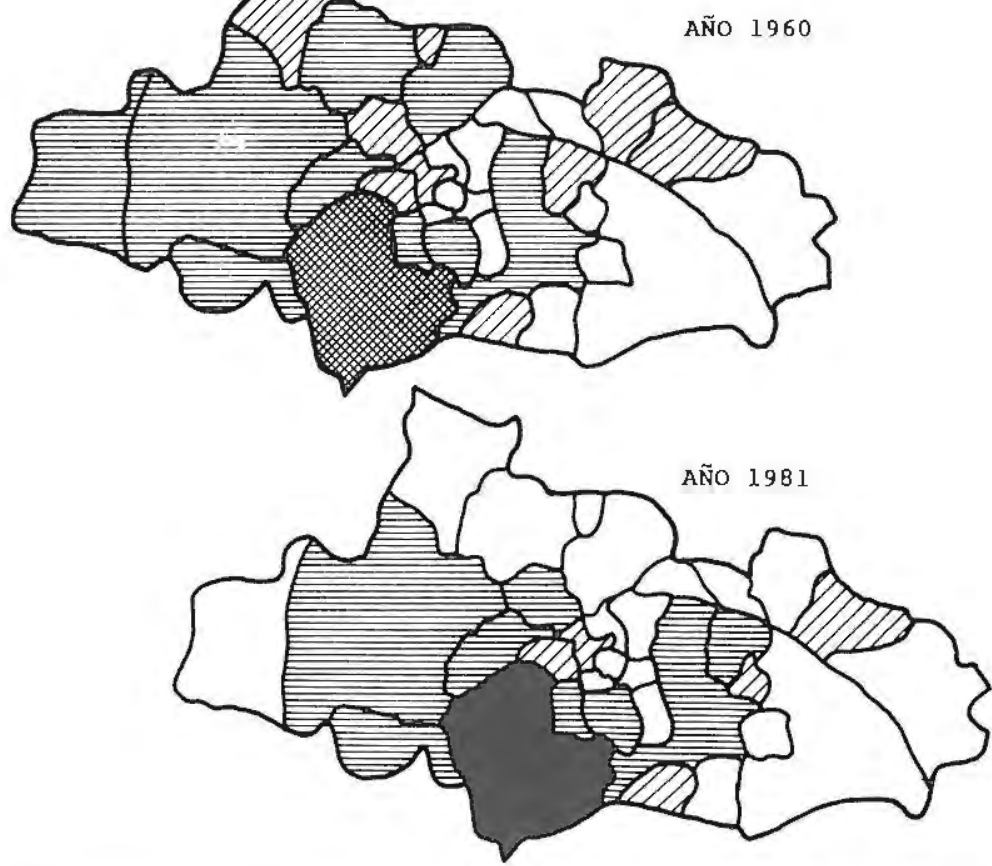

$\square$ De 0 a $0^{\prime} 01$

ED De $0^{\circ} 01$ a $0^{\prime} 1$

De 0.1 a 1

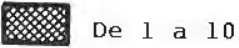

De 10 a 20

Fuente: I.N.E.

J. Monteagudo. 
CUADRO IY

DISPERSION DEL HABITAT EN LA SIERRA DE HUELVA. INDICE DE COLAS (*)

1981

\begin{tabular}{|c|c|c|c|c|c|c|c|c|c|c|c|c|c|c|c|c|}
\hline \multirow{2}{*}{ Municipios } & \multirow{2}{*}{$\mathrm{S} .\left(\mathrm{km}^{2}\right)$} & \multicolumn{5}{|c|}{1940} & \multicolumn{5}{|c|}{1960} & \multicolumn{5}{|c|}{1981} \\
\hline & & $\mathrm{T}$. & C. & E. & N. & I. & T. & C. & E. & N. & I. & $\mathrm{T}$ & C. & E. & N. & I. \\
\hline 1. Alájar & 41,9 & 1.921 & 1.501 & 420 & 9 & 0,251 & 1.540 & 1.278 & 262 & 9 & 0,295 & 912 & 874 & 38 & 5 & 0,240 \\
\hline 3. Aracena & 180,0 & 7.737 & 5.668 & 2.069 & 17 & 0,540 & 7.643 & 5.605 & 2.038 & 17 & 0,546 & 6.328 & 5.260 & 1.068 & 6 & 0,205 \\
\hline 4. Aroche & 498,4 & 6.771 & 5.659 & 1.112 & 8 & 0,705 & 6.686 & 5.319 & 1.367 & 9 & 0,843 & 3.933 & 3.497 & 436 & 7 & 0,998 \\
\hline 5. Arroyomolinos de León & 91,7 & 2.358 & 2.245 & 113 & 2 & 0,082 & 2.190 & 2.102 & 88 & 2 & 0,087 & 1.268 & 1.268 & 0 & 0 & 0,000 \\
\hline 8. Cañaveral de León & 45,2 & 985 & 910 & 75 & 9 & 0,447 & 954 & 954 & 0 & 0 & 0,000 & 572 & 572 & 0 & 0 & 0,000 \\
\hline 9. Castaño del Robledo & 12,8 & 573 & 488 & 85 & 2 & 0,052 & 429 & 429 & 0 & 0 & 0,000 & 230 & 230 & 0 & 0 & 0,000 \\
\hline 10. Corteconcepción $\ldots \ldots \ldots$ & 48,6 & 1.283 & 595 & 688 & 7 & 0,572 & 1.179 & 704 & 475 & 1 & 0,069 & 748 & 460 & 288 & 1 & 0,106 \\
\hline 11. Cortegana & 173,1 & 7.179 & 4.688 & 2.491 & 13 & 0,480 & 8.344 & 5.171 & 3.173 & 13 & 0,435 & 5.548 & 4.054 & 1.494 & 5 & 0,213 \\
\hline 12. Cortelazor & 39,1 & 685 & 640 & 45 & 3 & 0,183 & 608 & 608 & 0 & 0 & 0,000 & 422 & 422 & 0 & 0 & 0,000 \\
\hline 13. Cumbres de Enmedio & 13,7 & 235 & 207 & 28 & 1 & 0,066 & 218 & 206 & 12 & 1 & 0,067 & 71 & 71 & 0 & 0 & 0,000 \\
\hline 14. Cumbres de San Bartolomé ... & . 143,4 & 1.516 & 1.331 & 185 & 16 & 1,724 & 1.591 & 1.182 & 409 & 3 & 0,364 & 728 & 728 & 0 & 0 & 0,000 \\
\hline 20. Higuera de la Sierra & 23,8 & 2.135 & 2.094 & 41 & 5 & 0,057 & 1.696 & 1.696 & 0 & 0 & 0,000 & 1.320 & 1.320 & 0 & 0 & 0,000 \\
\hline 21. Hinojales & 19,0 & 1.151 & 1.145 & 5 & 3 & 0,050 & 1.007 & 1.007 & 0 & 0 & 0,000 & 509 & 509 & 0 & 0 & 0,000 \\
\hline$\ldots$ & 25,1 & 3.540 & 1.842 & 1.698 & 5 & 0,068 & 3.376 & 1.597 & 1.779 & 4 & 0,063 & 2.454 & 1.324 & 1.130 & 3 & 0,057 \\
\hline 23. Linares de la Sierra ................ & 27,9 & 758 & 729 & 29 & 5 & 0,191 & 601 & 601 & 0 & 0 & 0,000 & 310 & 310 & 0 & 0 & 0,000 \\
\hline 24. Marines, Los & 9,9 & 596 & 586 & 10 & 8 & 0,135 & 628 & 628 & 0 & 0 & 0,000 & 380 & 380 & 0 & 0 & 0,000 \\
\hline 25. Nava, La & 59,8 & 823 & 541 & 282 & 2 & 0,221 & 1.038 & 647 & 391 & 2 & 0,185 & 390 & 372 & 18 & 1 & 0,161 \\
\hline 26. Puerto Moral & 19,6 & 331 & 313 & 18 & 10 & 0,626 & 297 & 297 & 0 & 0 & 0,000 & 219 & 209 & 10 & 1 & 0,094 \\
\hline 27. Rosal de la Frontera & 210,3 & 3.466 & 2.845 & 621 & 6 & 0,444 & 3.150 & 2.839 & 311 & 3 & 0,222 & 1.930 & 1.930 & 0 & 0 & 0,000 \\
\hline 28. Santa Ana la Real & 26,8 & 1.164 & 566 & 598 & 3 & 0,142 & 923 & 461 & 462 & 3 & 0,174 & 614 & 402 & 212 & 3 & 0,200 \\
\hline 29. Santa Ollalla del Cala .......... & 195,9 & 3.757 & 3.545 & 212 & 11 & 0,608 & 4.630 & 4.630 & 0 & 0 & 0,000 & 2.204 & 2.204 & 0 & 0 & 0,000 \\
\hline 30. Valdelarco & 14,1 & 765 & 76.5 & 0 & 0 & 0,000 & 638 & 638 & 0 & 0 & 0,000 & 319 & 319 & 0 & 0 & 0,000 \\
\hline 31. Zufre & 333,6 & 2.955 & 2.013 & 942 & 20 & 3,314 & 2.491 & 2.491 & 0 & 0 & 0,000 & 1.820 & 1.820 & 0 & 0 & 0,000 \\
\hline
\end{tabular}

Fuente: Nomenclátor de 1940, 1960 y 1981 (I.N.E.). Elaboración propia.

$\left(^{*}\right) \mathrm{I}=$ S.N/T-E. S: Superficie municipal; N: N. ${ }^{\circ}$ núcleos en disperso; T: Población total; E: Población en disperso; C: Población núcleo princìpal (T-E). 
En 1981 se agudiza el proceso de concentración del habitat. Almonaster vuelve a superar el índice 10, debido en este caso a que disminuye la población de la cabecera municipal que representa el denominador, a pesar de que el número de núcleos dispersos se reduce a 20. En 1981 ningún municipio excepto éste tiene 10 núcleos dispersos, y 26 tienen menos de 5 núcleos, de los que 17 sólo tienen población en la cabecera.

Es evidente que la Sierra no sólo pierde población, sino que además esa población abandona principalmente los núcleos más pequeños produciendo una progresiva concentración. Las repercusiones directas, dado el carácter agropecuario de la economía y la dificultad de las comunicaciones, son el alejamiento de la explotación y en algunos casos el propio abandono de la misma.

\section{CONCLUSIONES}

La Sierra de Huelva es una de las comarcas más deprimidas de Andalucía. Su actividad, eminentemente agropecuaria y forestal, no le permite sostener el crecimiento vegetativo de su población y se produce un éxodo masivo. Ese éxodo ha estado potenciado por la absorción que produce, dentro de su propia provincia, el sector industrial de la capital y su entorno, y por el abandono sistemático al que ha estado sometida por parte de las administraciones nacional y provincial, y en la actualidad también autonómica (falta de carreteras, deficiencia de las existentes, falta de servicios sociales, etc.). Su despoblación trae como consecuencia, no sólo la progresiva desertización y abandono de núcleos de población y explotaciones, sino también el envejecimiento progresivo de la población que permanece, reduciéndose e incluso anulándose el crecimiento natural y creando un círculo de pobreza-emigración-pobreza.

Es por tanto una de las comarcas que reclaman atención preferente, puesto que de continuar esta línea quedará convertida en un auténtico desierto, y paradójicamente ni una sola de sus hectáreas había sido incluida en la delimitación de áreas de montaña. 


\section{BIBLIOGRAFIA}

AVILA FERNANDEZ, D.: Campofrío. Una forma de vida entre la Sierra y la mina. I.E.O., Huelva, 1981.

- Ocupación, usos y organización del espacio productivo en la Sierra de Huelva. Tesis doctoral, Dtpo. de Geografía, Univ. de Sevilla, 1982. Inédita.

- "La floración de los matorrales, un recurso natural de Sierra Morena". Revista de Estudios Andaluces, 3, pp. 145-150, 1984 .

BANESTO: Anuario del Mercado Español. Varios años. Esp. 1984.

BENABENT, M.: "Ordenación del territorio, planificación y gestión de las áreas de montaña", Revista de Estudios Andaluces, 6, pp. 65-74, 1986.

BOSQUE MAUREL, J.: "Factores geográficos en el desarrollo de Andalucía", en Estudio socioeconómico de Andalucia, con KADE, LINZ y KOTTER. Inst. Des. Ec., Madrid, 1970.

BOSQUE MAUREL, J. (Dir.): Estructura económica de Andalucía. Cámaras Oficiales de Comercio, Ind. y Nav. de Andalucía, 1978.

CAMARA OFICIAL DE COMERCIO, INDUSTRIA Y NAVEGACION DE HUELVA: Memoria. Varios años, última 1984, Huelva, 1985.

CANO GARCIA, G.: "Unidad y diversidad de la geografía andaluza". Revista de Estudios Andaluces, 1, pp. 9-22, 1983.

- "Comarcalización y Reforma Agraria en Andalucía”. Rev. Estudios Andaluces, 3, pp. 101-124, 1984.

COLAS, R.: "Repartition de l'habitat". Bol. A.G.F., 1945.

DELGADO CABEZA, M.: Dependencia y marginación de la economía andaluza. Monte de Piedad y Caja de Ahorros de Córdoba, 1981.

DIAZ DEL OLMO, F.: "Sierra Morena. Nuevas consideraciones en sus interpretaciones de geomorfología y geología regional". Rev. Est. Andaluces, 1, pp. 35-42, 1983.

FOURNEAU, F.: El impacto del Polo de Desarrollo en la provincia de Huelva (1964-1974). I.D.R., Univ. Sevilla, 1978.

- La provincia de Huelva y los problemas del desarrollo regional. I.E.O., Huelva, 1983.

GARCIA BARBANCHO, A.: La población andaluza. I.D.R., Granada, 1980.

- "Las comarcas en el desarrollo económico español", I1 reunión de estudios regionales, Madrid, 1976.

- "La población" en Decadencia y crisis en Andalucia. Dir. VELARDE FUERTES, J. I.D.R., Univ. Sevilla, 1982.

GIL VARON, L.: Minería y migraciones. Riotinto, 1873-1973. Córdoba, 1984, ed. el autor.

I.N.E.: Censo de Población de 1981. Tomo III. Resultados provinciales: Huelva. Madrid, 1984. Tomo IV. Resultados municipales. Vol. I. Huelva, 1985. Tomo I. Poblaciones de hecho y de derecho de los municipios españoles. Madrid, 1982.

JORDA BORRELL, R.: Dinámica y distribución recientes de la población andaluza. I.D.R., Univ. Sevilla, 1985. 
JUNG, J.: La ordenación del espacio rural. I.E.A.L., Madrid, 1972.

LEGUINA, J. y NAREDO, J.M.: "Exodo rural y envejecimiento de la población activa agraria", Inf. Com. Esp., 1974, 496.

MARCHENA GOMEZ, M.: La distribución de la población en Andalucía, 1960-1981. Dip. Prov., Univ. Sevilla, 1984.

MARQUEZ DOMINGUEZ, J.: La nueva agricultura onubense: protagonismo agrario del municipio de Moguer. I.D.R., Univ. Sevilla, 1986.

MARQUEZ FERNANDEZ, D.: La geoeconomía forestal de Huelva y el dilema de sus eucaliptales. I.D.R., Univ. Sevilla, 1977.

- "Las repoblaciones de eucaliptos y su impacto en la última década 1973-1983". Revista de Estudios Andaluces, 5, 1985, pp. 135-142.

MONTEAGUDO LOPEZ-MENCHERO, J.: Evolución geodemográfica de un sector periurbano de Huelva: Palos de la Frontera y Moguer, 1960-1975. Col. Univ. Rábida, Huelva, 1980.

- "La ciudad de Huelva: resultado demográfico de la polarización industrial y la despoblación rural". III Coloquio Ibérico de Geografía, Barcelona, 1984.

- "La actual organización del territorio en la provincia de Huelva". III Coloquio Ibérico de Geografía, Barcelona, 1984.

MORENO ALONSO, M.: La vida rural en la Sierra de Alajar. Caja Rural Provincial, Huelva, 1981. MYRDAL, G.: Teoría económica y regiones subdesarrolladas. F.C.E., México, 1959.

PUYOL ANTOLIN, R.: Emigración y desigualdades regionales en España. E.M.E.S.A., Madrid, 1975.

ROUX, B.: Crisis agraria en la Sierra Andaluza. I.D.R., Univ. Sevilla, 1975.

- "La crise económique actuelle dans la Sierra de Aracena". Melanges de la Casa de Velázquez, X, 1974.

RUBIO RECIO, J.M.: "Paisajes vegetales de Sierra Morena occidental y su utilización”. Gades, 7, 1981.

SANTOS BRAVO, N.: Alajar, un municipio de la Sierra de Aracena (Huelva). Memoria de Licenciatura, Univ. Sevilla. Inédito, 1974.

SUAREZ JAPON, J.M.: El habitat rural en la Sierra de Cádiz. Dip. provincial, Cádiz, 1982.

TORRE, A. de la: Política de empleo en Andalucía. Factores demográficos y económicos. I.D.R., Universidad de Sevilla, 1985. 